\title{
Dimension dependence of correlation energies in two-electron atoms
}

\author{
J. G. Loeser \\ Department of Chemistry and Society of Fellows, University of Michigan, Ann Arbor, Michigan 48109- \\ 1055 \\ D.R. Herschbach \\ Department of Chemistry, Harvard University, Cambridge, Massachusetts 02138
}

(Received 11 August 1986; accepted 9 December 1986)

\begin{abstract}
Correlation energies (CEs) for two-electron atom ground states have been computed as a function of the dimensionality of space $D$. The classical limit $D \rightarrow \infty$ and hyperquantum limit $D \rightarrow 1$ are qualitatively different and especially easy to solve. In hydrogenic units, the CE for any two-electron atom is found to be roughly $35 \%$ smaller than the real-world value in the $D \rightarrow \infty$ limit, and about $70 \%$ larger in the $D \rightarrow 1$ limit. Between the limits the $C E$ varies almost linearly in $1 / D$. Accurate approximations to real CEs may therefore be obtained by linear interpolation or extrapolation from the much more easily evaluated dimensional limits. We give two explicit procedures, each of which yields CEs accurate to about $1 \%$; this is comparable to the best available configuration interaction calculations. Steps toward the generalization of these procedures to larger atoms are also discussed.
\end{abstract}

\section{INTRODUCTION}

Most electronic structure calculations performed today employ the self-consistent field (SCF) method, and therefore omit the correlation energy. ${ }^{1}$ In the SCF approach each electron is assumed to move independently in the average field of the other electrons. Each electron is described by an orbital, or one-electron wave function, and the system as a whole is described by a configuration, or assignment of electrons to orbitals. The correlation energy (CE) is the difference between the exact energy (excluding relativistic effects) and the optimal SCF energy, which is the HartreeFock (HF) limit. The CE is typically a small fraction of the total energy, but of the same order of magnitude as the energies involved in chemical phenomena. Depending on the application, therefore, it may or may not be safe to ignore it. If the electronic state does not change much, as in the optimization of a molecular geometry, SCF calculations will often suffice. On the other hand, the CE will usually be a major factor in phenomena where the electronic state does change, such as ionizations and dissociations. ${ }^{2}$

The main reason that most calculations are performed at the SCF level is simply that CEs are very difficult to calculate. In the most common procedure, the single configuration of the SCF wave function is combined with a number of others. Although this method of configuration interaction (CI) can in principle yield the full correlation energy, in practice it requires a very large number of configurations to do a good job. This is because the "correlation holes" in the exact wave functions created by the instantaneous electronelectron repulsions are very difficult to model using a basis of SCF wave functions. ${ }^{1}$

Quantitative knowledge of atomic CEs remains surprisingly limited. Although accurate SCF wave functions and energies have been computed for all atoms, ${ }^{3}$ approximate CEs are known only as far as argon. CI calculations have been performed for first row atoms (up to $Z=10$ ). ${ }^{4}$ The errors, due to incompleteness of the basis set, are $3 \%-5 \%$. For second row atoms (up to $Z=18$ ), CEs have been obtained by utilizing experimental data in conjuction with SCF calculations. ${ }^{5}$ The uncertainties, which in this case are due to the relativistic corrections, are $5 \%-10 \%$. For larger atoms (beyond $Z=18$ ) the only available CEs are based either on extrapolation from values for smaller atoms, ${ }^{6}$ or else on approximations such as the electron gas model. ${ }^{7}$ The values yielded by these two procedures differ by a factor of 2 or more. ${ }^{6,8}$

In this paper we examine a new approach to atomic correlation energies which exploits dimensional continuation methods recently applied to two-electron atoms. ${ }^{9-16}$ The motivating idea is to generalize to spaces of dimensionality $D \neq 3$, do calculations wherever they are easy, and then work back to $D=3$. Two cases in which many problems can be expected to simplify are $D=1$ and the $D \rightarrow \infty$ limit. For Coulomb problems the first of these is more correctly designated the $D \rightarrow 1$ limit, and is even easier to solve than might be anticipated from the restriction to one degree of freedom. The simplest dimensional variant, however, will generally be the $D \rightarrow \infty$ limit.

In Sec. II we examine the nature of the limits $D \rightarrow \infty$ and $D \rightarrow 1$, and give explicit results for two-electron atom CEs in these limits. Section III presents the results for other dimensions, as revealed by computer calculation. In Sec. IV we explore the physics underlying the observed behavior, and in Sec. V utilize this to motivate two very simple methods for extracting real-world CEs from the dimensional limits. We also show why these two schemes work so much better than the customary approach used with dimensional continuation, namely the $1 / D$ expansion. ${ }^{9-12}$ Finally, in Sec. VI we discuss briefly the encouraging prospects for generalization of these methods to larger atoms.

\section{DIMENSIONAL LIMITS}

We consider the ground state of a two-electron atom of nuclear charge $Z$ in a space of dimensionality $D$. We assume a Hamiltonian identical in form to that for $D=3$, but with Laplacians and Euclidean distances now understood to be those appropriate to $D$-dimensional space. $D \rightarrow \infty$ and $D \rightarrow 1$ constitute singular limits for this problem. In order to obtain 
finite atomic energies and sizes in the two limits, we employ scaled variables. Appropriate units are $[2 Z /(D-1)]^{2}$ hartree for energies and $D(D-1) / 2 Z$ bohr radii for distances. The $D$-dimensional Hamiltonian in these units is

$$
\begin{aligned}
H_{D}= & -\frac{1}{2} \delta^{2}\left[\nabla_{1}^{2}+\nabla_{2}^{2}\right]+\frac{1}{2}(1-\delta) \\
& \times\left[-\frac{1}{\rho_{1}}-\frac{1}{\rho_{2}}+\frac{\lambda}{\rho_{12}}\right],
\end{aligned}
$$

with $\delta=1 / D$ and $\lambda=1 / Z$. We observe that the coefficient of the kinetic terms vanishes in the limit $D \rightarrow \infty$, and that of the potential terms in the limit $D \rightarrow 1$. However, careful examination of the limits reveals that in neither case do the terms simply drop out.

\section{A. The classical limit, $D \rightarrow \infty$}

The reason that the Laplacians in $H_{D}$ do not drop out in the $D \rightarrow \infty$ limit is that they give rise to terms of order $D^{2}$ which cancel out the coefficient $1 / D^{2}$. These contributions come from centrifugal terms having $D^{2}$ in place of the usual $L^{2}$, and arise because the $D$-dimensional volume element works against "tight" configurations in the same way that angular momentum does. The volume element is $\rho_{1}^{D-1} \rho_{2}^{D-1} \sin ^{D-2} \theta$, where the three variables are the lengths of the two electron-nucleus vectors and the angle between them. When this is incorporated to simplify the Laplacians, the terms in the limiting Hamiltonian which survive constitute an effective potential, which for $S$ states is

$$
V_{\infty}=\frac{1}{8}\left[\frac{1}{\rho_{1}^{2}}+\frac{1}{\rho_{2}^{2}}\right] \frac{1}{\sin ^{2} \theta}+\frac{1}{2}\left[-\frac{1}{\rho_{1}}-\frac{1}{\rho_{2}}+\frac{\lambda}{\rho_{12}}\right] .
$$

The minimum of this potential defines the exact solution for the $D \rightarrow \infty$ limit. $^{-12}$ This localization into a well-defined configuration is quite general and is the reason for calling $D \rightarrow \infty$ a classical limit.

In this case the limiting configuration has the two electrons at fixed distances from the nucleus, with an angle somewhat greater than $90^{\circ}$ (and increasing with $\lambda$ ) between them. For $\lambda<\lambda_{0} \doteq 0.81439$, the configuration has isosceles symmetry, and the exact energy may be evaluated explicitly as ${ }^{15}$

$\epsilon_{\infty}=-1-\frac{5}{32} \lambda^{2}+\frac{1}{2048}\left[\lambda^{4}+\lambda\left(\lambda^{2}+128\right)^{3 / 2}\right]$.

For $\lambda>\lambda_{0}$, and in particular for the hydride ion, the minimum energy configuration is asymmetric, with one electron closer to the nucleus than the other; Eq. (3) no longer gives the minimum energy but rather corresponds to a saddle point between two equivalent minima.

In the Hartree-Fock approximation the assumed form for the wave function precludes any explicit $\theta$ dependence. The angle is therefore determined solely by a geometrical factor, the term $\sin ^{D-2} \theta$ in the volume element. This fixes the angle at $90^{\circ}$. Minimization of the effective potential under this constraint gives ${ }^{17}$

$$
\epsilon_{\infty}^{\mathrm{HF}}=-1+\frac{1}{\sqrt{2}} \lambda-\frac{1}{8} \lambda^{2}
$$

This pertains to $\lambda<\lambda_{0}^{\mathrm{HF}} \doteq 1.13137$, where the minimum configuration has isosceles symmetry.

The CE in the $D \rightarrow \infty$ limit, $\Delta \epsilon_{\infty}$, is now given by the difference of Eqs. (3) and (4). For the neutral atom $(\lambda=1$ / 2 ) this is about $0.99 \%$ of the total energy, distinctly smaller than the corresponding $D=3$ value of $1.45 \%$. Although these percentages vary somewhat with the nuclear charge, their ratio remains fairly constant (between 0.67 and 0.69 ) over the full range of two-electron atoms.

\section{B. The hyperquantum limit, $D \rightarrow 1$}

In this limit no terms in the potential survive except perhaps when an interparticle coordinate vanishes, thereby causing one of the Coulomb terms to diverge. Care in taking the limit shows that each potential term is in fact a delta function of unit strength, so that the $D \rightarrow 1$ limit of the Hamiltonian is ${ }^{18}$

$$
H_{1}=-\frac{1}{2}\left[\frac{\partial^{2}}{\partial \rho_{1}^{2}}+\frac{\partial^{2}}{\partial \rho_{2}^{2}}\right]-\delta\left(\rho_{1}\right)-\delta\left(\rho_{2}\right)+\lambda \delta\left(\rho_{12}\right) \text {. }
$$

A single electron in a delta function potential has only one bound state, and this is classically forbidden except at the singularity. Since $H_{1}$ describes two such electrons perturbed only by a purely repulsive interelectron term, it has at most one bound state, and this is classically forbidden except on a set of measure zero $\left(\rho_{1} \rho_{2}=0\right)$. It is thus appropriate to call $D \rightarrow 1$ a hyperquantum limit.

The $D \rightarrow 1$ limit is not quite as easy to solve as the $D \rightarrow \infty$ limit, but is still much simpler than the $D=3$ problem for two reasons. First, the restriction of the physics to a line eliminates all angular variables. Thus, $H_{1}$ depends only on the two radial coordinates. Second, the delta functions simplify the solution of the Schrödinger equation. For the twoelectron atom this allows both the exact and HF versions of the problem to be reduced to one variable. The exact solution has been obtained ${ }^{19}$ as a series expansion,

$$
\begin{aligned}
\epsilon_{1}= & -1+\frac{1}{2} \lambda-\left[\frac{3}{8}-\frac{2}{3 \pi}\right] \lambda^{2} \\
& +\left[\frac{1}{6 \pi}-\frac{5}{128}\right] \lambda^{3}-\cdots,
\end{aligned}
$$

whereas the HF solution has been obtained explicitly, ${ }^{20}$

$$
\epsilon_{1}^{\mathrm{HF}}=-1+\frac{1}{2} \lambda-\frac{1}{12} \lambda^{2} .
$$

The CE in the $D \rightarrow 1$ limit, $\Delta \epsilon_{1}$, is now given by the difference between Eqs. (6) and (7). In this case the CE for the neutral atom $(\lambda=1 / 2)$ is about $2.28 \%$ of the total energy, considerably larger than the $D=3$ value. In fact, the ratio $\Delta \epsilon_{1} / \Delta \epsilon_{3}$ falls between 1.70 and 1.72 for almost all two-electron atoms.

\section{Dependence on nuclear charge}

Upon taking the difference of either Eqs. (3) and (4) or Eqs. (6) and (7) to obtain the CE, the terms of order $\lambda^{\circ}$ and $\lambda^{1}$ cancel, so the CE begins at order $\lambda^{2}$. That this is a general result, independent of $D$, may be seen as follows: For $\lambda \rightarrow 0$ the $D$-dimensional Hamiltonian $H_{D}$ in Eq. (1) is separable, 
so the exact and $\mathrm{HF}$ wave functions must be identical in this limit. The corresponding energies will therefore agree to first order in $\lambda$, and their difference will vanish to that order.

\section{CALCULATIONS FOR $1<D<\infty$}

To examine two-electron atom CEs between the dimensional limits, we have utilized computer programs which generate accurate approximations to the exact and HF energies for arbitrary values of $D$. The algorithms were obtained by generalizing appropriate three-dimensional methods, namely the precise variational approach pioneered by $\mathrm{Hyl}$ leraas and perfected by Pekeris, and the Hartree-FockRoothaan algorithm. These generalizations have been presented elsewhere. ${ }^{16}$

CEs were computed for a wide range of spatial dimensionalities $D$ and nuclear charges $Z$. The problem can be formulated for arbitrary real values of these parameters, but we consider here only the domain $1<D<\infty, 1<Z<\infty$. ( $T$ wo-electron atoms are unbound for $Z$ much less than unity, formally unnormalizable for $D$ less than unity, ${ }^{18}$ and computationally difficult in either case. ${ }^{16}$ ) Table I gives CEs for selected values of $D$ and $Z$.

We view the $C E$ as a function of the inverse parameters $\delta=1 / D$ and $\lambda=1 / Z$. As we see from Eq. (1), these are natural perturbation parameters for the problem, $\delta$ measuring the departure from the classical limit and $\lambda$ measuring the strength of the interelectron repulsion. Also, these parameters collapse the domain of interest to a finite one, $0<\delta<1,0<\lambda<1$. Figure 1 gives a contour plot of the $\mathrm{CE}$ as a function of $\delta$ and $\lambda$. (Because of the basic $\lambda^{2}$ dependence of the $\mathrm{CE}$, the values have been normalized by $\lambda^{2}$. Values are not plotted for combinations of the parameters which yield no bound state, because CEs cannot be defined there.)

Figure 1 reveals a variation of the $C E$ with $\delta$ and $\lambda$ which is surprisingly regular, considering the range of physics involved. The variation is monotonic, and in fact quite linear, with respect to each parameter. To large extent the two functional dependencies factor, and we consider them separately.

For any fixed value of the dimensionality, the variation with respect to $\lambda$ of the CE as plotted in Fig. 1 is quite modest. Its magnitude decreases with $\lambda$, but in general by only about $25 \%$ between $\lambda=0$ and $\lambda=1$. This generalizes the observation that the CEs of real two-electron atoms are all roughly the same as that of helium, though they do increase slightly with nuclear charge. ${ }^{21}$

Our main concern in this paper is the variation of the CE with respect to $\delta$. This is more pronounced than that with respect to $\lambda$, but also more regular. In Fig. 2 the CEs are plotted as a function of $\delta$ for several values of the nuclear charge. Each curve is normalized by the $D \rightarrow \infty$ value. We see that in each case the CE increases almost linearly with $\delta$ by a factor of roughly 2.5 between the $D \rightarrow \infty$ and $D \rightarrow 1$ limits.

To quantify the linearity, we consider the vertical deviations of each curve from a straight line drawn between its endpoints. These nonlinearities are plotted in Fig. 3. They are most pronounced for high $D$ and low $Z$. This is the domain of weakly bound states adjoining the blank region of

TABLE I. Correlation energies for D-dimensional two-electron atom ground states."

\begin{tabular}{|c|c|c|c|c|c|c|}
\hline$D$ & $Z=1$ & $Z=1.2$ & $Z=1.5$ & $Z=2$ & $Z=3$ & $Z=6$ \\
\hline 1 & -0.063877 & -0.046307 & -0.030849 & -0.0180149 & -0.0082910 & -0.00214147 \\
\hline $12 / 11$ & -0.0605139 & & -0.0292528 & -0.0170941 & -0.0078735 & -0.00203531 \\
\hline $6 / 5$ & -0.0571698 & -0.0414526 & -0.0276377 & -0.0161587 & -0.0074479 & -0.00192679 \\
\hline $4 / 3$ & -0.0538753 & & -0.0260128 & -0.0152121 & -0.00701520 & -0.001816150 \\
\hline $3 / 2$ & -0.0506676 & -0.0366208 & -0.0243877 & -0.0142591 & -0.00657748 & -0.001703786 \\
\hline $12 / 7$ & -0.0475924 & & -0.0227740 & -0.01330555 & -0.00613718 & -0.001590290 \\
\hline 2 & -0.04467 & -0.03197358 & -0.02118450 & -0.012358400 & -0.005697353 & -0.0014764308 \\
\hline $12 / 5$ & & & -0.019631523 & -0.0114248797 & -0.0052614984 & -0.0013631469 \\
\hline 3 & & -0.02768900 & -0.0181231510 & -0.01051109536 & -0.00483313458 & -0.00125149239 \\
\hline 4 & & & -0.0166543979 & -0.009618568475 & -0.004414782700 & -0.001142473112 \\
\hline $24 / 5$ & & & & -0.009177769781 & -0.004209360445 & -0.0010891667234 \\
\hline 6 & & -0.0237 & -0.0151865323 & -0.008736537476 & -0.004005688625 & -0.0010366661320 \\
\hline 8 & & & -0.0144190659 & -0.008288641631 & -0.003802358950 & -0.0009848453409 \\
\hline $48 / 5$ & & & & -0.008059078454 & -0.003700092198 & -0.0009591074372 \\
\hline 12 & & & -0.0135873412 & -0.007823282359 & -0.003596847433 & -0.0009334143626 \\
\hline 16 & & & -0.0131276444 & -0.007578586023 & -0.003492030723 & -0.0009076928548 \\
\hline $96 / 5$ & & & & -0.007451807533 & -0.003438800927 & -0.0008947932118 \\
\hline 24 & & & -0.0126199591 & -0.007321327916 & -0.003384873469 & -0.0008818498739 \\
\hline 32 & & & -0.0123407635 & -0.007186444327 & -0.003330115684 & -0.0008688476824 \\
\hline $192 / 5$ & & & & -0.007117090292 & -0.003302378758 & -0.0008623193630 \\
\hline 48 & & & -0.0120386815 & -0.007046298865 & -0.003274375563 & -0.0008557699577 \\
\hline 64 & & & & -0.00697393003 & -0.003246083535 & -0.0008491971344 \\
\hline 96 & & & -0.0117068898 & -0.00689982623 & -0.003217478469 & -0.0008425984934 \\
\hline 192 & & & & -0.00682380918 & -0.003188534395 & -0.0008359715397 \\
\hline$\infty$ & & & -0.01133507635 & -0.006745675875 & -0.003159223481 & -0.0008293137160 \\
\hline
\end{tabular}

"Units are $[2 Z /(D-1)]^{2}$ hartree. Values were computed using generalizations to $D$-dimensional space of the Hylleraas-Pekeris and Hartree-Fock algorithms presented in Ref. 16. 


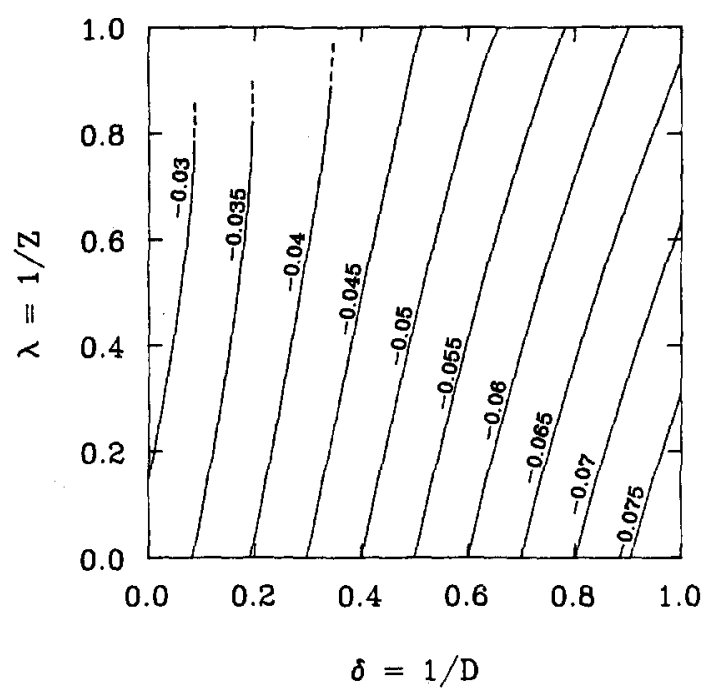

FIG. 1. Correlation energies for ground states of two-electron atoms as a function of the inverse spatial dimensionality $\delta=1 / D$ and inverse nuclear charge $\lambda=1 / Z$. Units are $[2 /(D-1)]^{2}$ hartree. Real atoms fall on the vertical section $\delta=1 / 3$ and neutral ones on the horizontal section $\lambda=1 / 2$. Atoms in the vicinity of the infinite-dimensional hydride ion (top left corner) have no bound states, so correlation energies cannot be calculated for them.

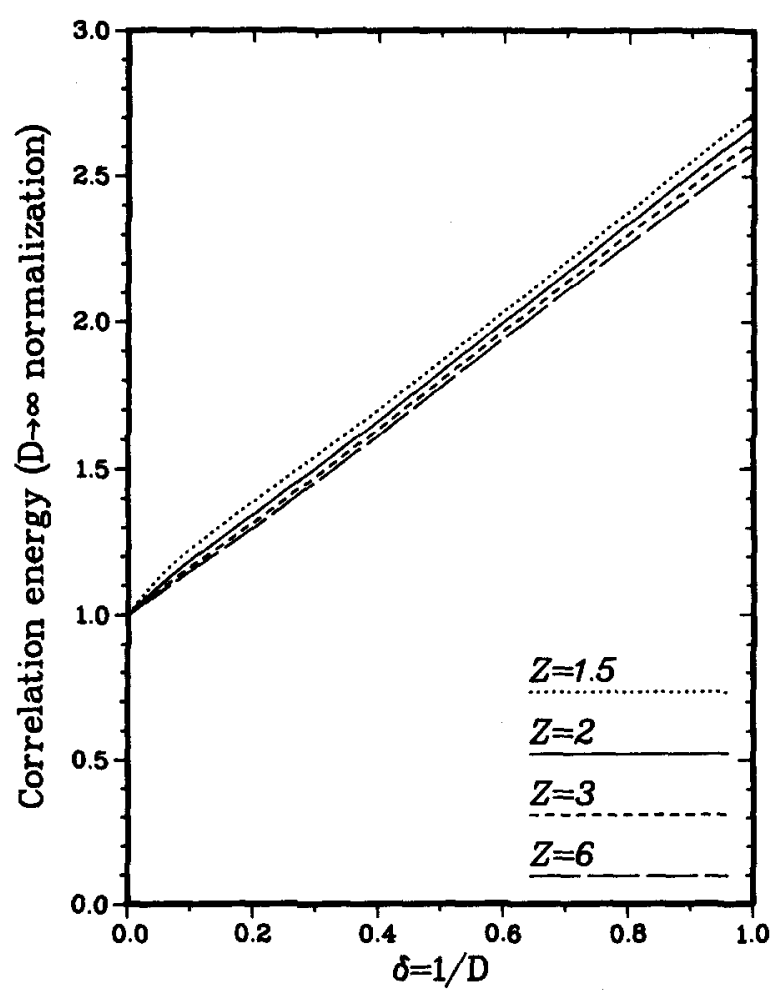

FIG. 2. Dimension-dependence of two-electron atom correlation energies for several values of the nuclear charge. Each curve is normalized by its $D \rightarrow \infty$ limit, which is the difference of Eqs. (3) and (4). Real atoms fall on the vertical section $\delta=1 / 3$. For $Z>2$ the curves are linear to about 1 part in 100. spontaneous ionization in Fig. 1. The focal point for the nonlinear behavior appears to be the symmetry breaking at $\delta=0, \lambda=\lambda_{0}$ mentioned above. This transition at $Z \doteq 1.23$ from a symmetric classical limit structure to an asymmetric one has its most significant effects at large but finite $D$. This may be seen by considering the expansion of the energy in powers of $\delta$ and $\lambda$. For small enough $\delta$ the energy is determined by the intercept ( the classical limit $D \rightarrow \infty$ ) and slope (the first-order semiclassical correction, or $1 / D$ term). The symmetry breaking has a very mild effect on the classical limit itself. The effect is proportional to $\left(\lambda-\lambda_{0}\right)^{2}$, which yields a discontinuity in the curvature at $\lambda_{0}$. The effect of the symmetry breaking on the semiclassical correction term is much more serious. ${ }^{15}$ Here it is proportional to $\left(\lambda-\lambda_{0}\right)^{1 / 2}$, which assumes infinite slope at $\lambda_{0}$. The effects of this term can be seen clearly in Fig. 3. As $Z$ is decreased, the initial slopes are increasingly dominated by the square root branch point at $Z \doteq 1.23$, and this appears to destroy the otherwise smooth behavior.

\section{ORIGINS OF LINEARITY}

We have seen that the deviations from linearity with respect to $1 / D$ become larger at small $Z$. On an absolute scale, however, they remain small. In fact, the nonlinearities remain less than $2 \%$ of the total correlation energy for all neutral atoms and positive ions $(1<D<\infty, 2<Z<\infty)$. We consider now the origins of this strikingly regular behavior.

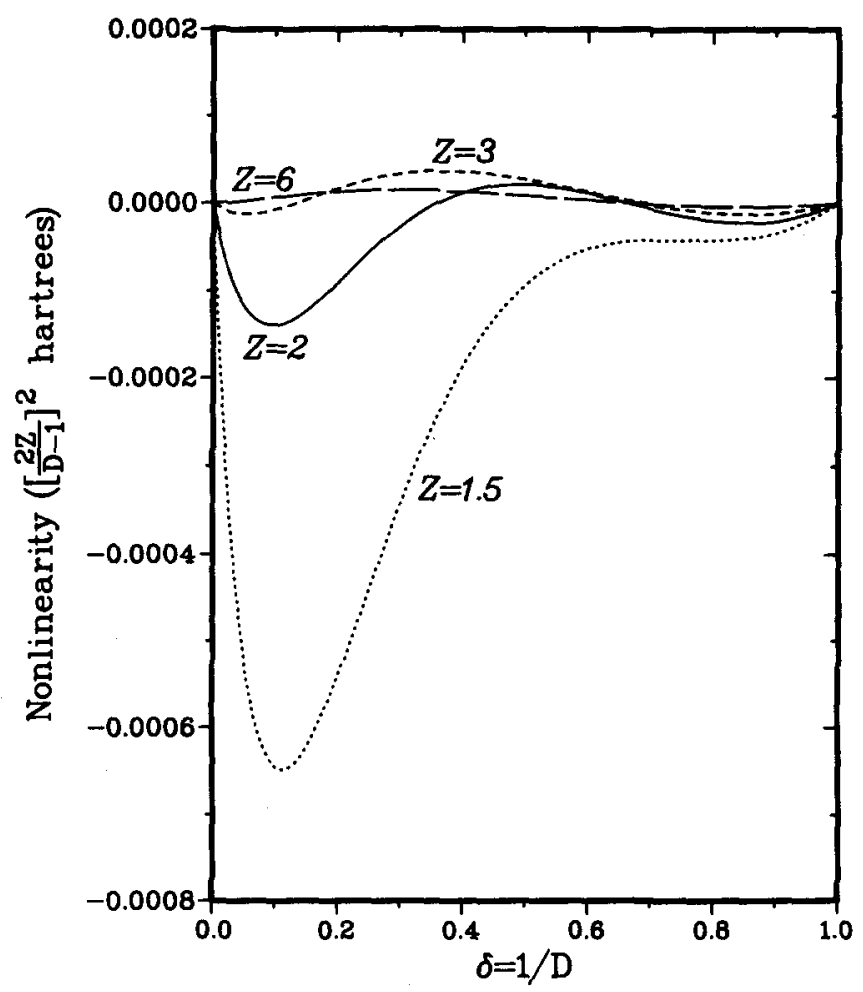

FIG. 3. Nonlinearities of the curves in Fig. 2 on an expanded scale. These are defined as the deviations of the curves from straight lines drawn between their endpoints (the classical limit $D \rightarrow \infty$ and the hyperquantum limit $D \rightarrow 1)$. Nonlinearities are most pronounced at high $D$ and small $Z$, which is the domain of weakly bound atoms. 
The CE for neutral atoms is plotted as a function of $\delta$ in Fig. 4(a), and the exact and HF energies (of which the CE is the difference) in Fig. 4(b). All three quantities show smooth and monotonic dependence on the dimensionality. As the difference of two much larger quantities, the CE might be expected to magnify any irregularities in the behavior of the total energies. As we have seen, however, the CE is highly linear.

The linearity results from a high degree of cancellation between the nonlinear components of the two total energies. This is made clear in Fig. 5, where the nonlinearities of the curves in Fig. 4 are plotted. Roughly speaking, the nonlinearities in the exact and HF energies cancel to 1 part in 100 . Since these were already only about $1 / 100$ of the total energies, the nonlinearities in the $\mathrm{CE}$ are a factor of $10^{4}$ smaller than the total energy.

\section{A. Perturbation analysis}

To understand this cancellation we turn to a perturbation treatment in $\lambda$. At the end of Sec. II, we noted that for any $D$ the exact and $H F$ wave functions agree to zeroth order in $\lambda$, and the corresponding energies to first order. In this simple observation we have an explanation for most of the cancellation observed in Fig. 5, because most of the nonlinearity in $\delta$ of the exact and HF energies is due to their common first-order term. It has been calculated explicitly as ${ }^{18}$

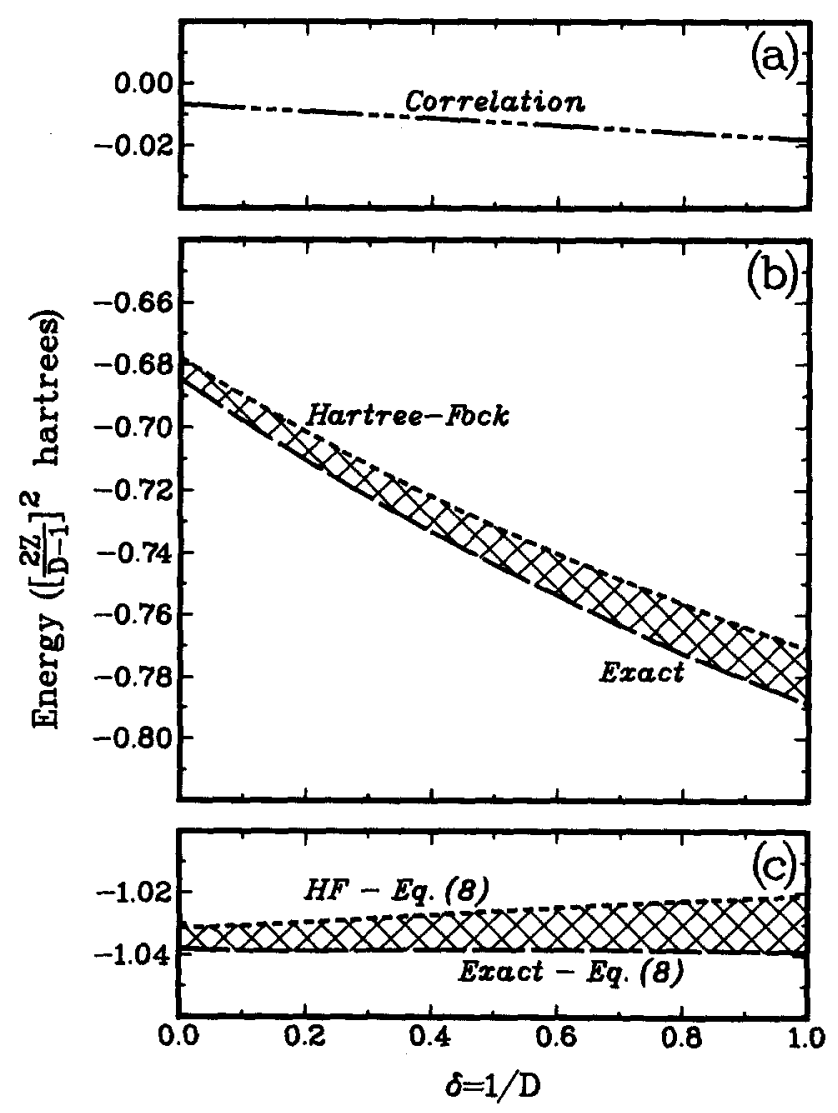

FIG. 4. Contributions to the energies of neutral two-electron atoms as a function of $\delta=1 / D$ : (a) the correlation energy; (b) exact and HartreeFock energies, with their difference (the correlation energy) shaded; (c) same, but with the first-order interelectron repulsion energy eliminated.

$$
\begin{aligned}
& \frac{\Gamma(D+1) \Gamma\left(\frac{1}{2} D+\frac{1}{2}\right)}{\Gamma\left(D+\frac{1}{2}\right) \Gamma\left(\frac{1}{2} D+1\right)} \lambda \\
& \quad=\frac{1}{\sqrt{2}}\left[1-\frac{3}{8} \delta+\frac{9}{128} \delta^{2}+\frac{39}{1024} \delta^{3}-\cdots\right] \lambda .
\end{aligned}
$$

It is the terms of order $\delta^{2}$ and higher which account for most of the bow in the two total energy curves. This may be seen by comparing the (b) and (c) panels of Figs. 4 and 5, which are identical except for the fact that the first-order term has been subtracted out in the (c) panels.

Comparing now panels (a) and (c), we see that there is still some cancellation of nonlinearities to be accounted for, so we turn to higher orders in $\lambda$. Explicit formulas analogous to Eq. (8) are no longer available, but accurate coefficients are available for several dimensionalities, ${ }^{15,18}$ and with these we can make a limited term-by-term assessment of linearity. This is done in Fig. 6. Dots indicate exact coefficients (solid for positive values and open for negative), and lines indicate coefficients linearly interpolated between the $D \rightarrow \infty$ and $D \rightarrow 1$ limit values; note that curvature in the lines is due solely to the use of a logarithmic scale. Table II gives the first six $\lambda$-expansion coefficients for the limiting cases, Table III those for $D=3$, and Table IV those for the two leading terms of the $1 / D$ expansion.

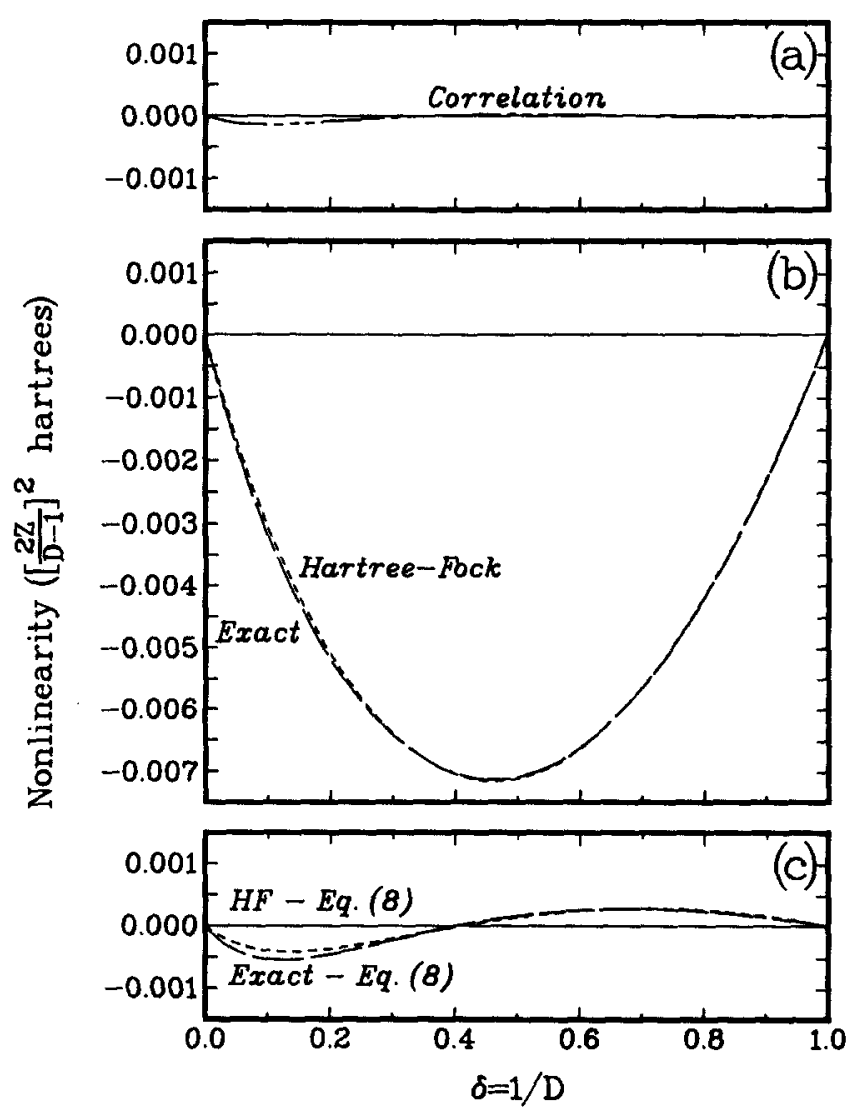

FIG. 5. Nonlinearities of the curves in Fig. 4 on an expanded scale. The near linearity of the correlation energy (a) results from cancellation of nonlinearities in the exact and HF energies (b). Most but not all of the nonlinearities which cancel may be attributed to the first-order interelectron repulsion term (c). 

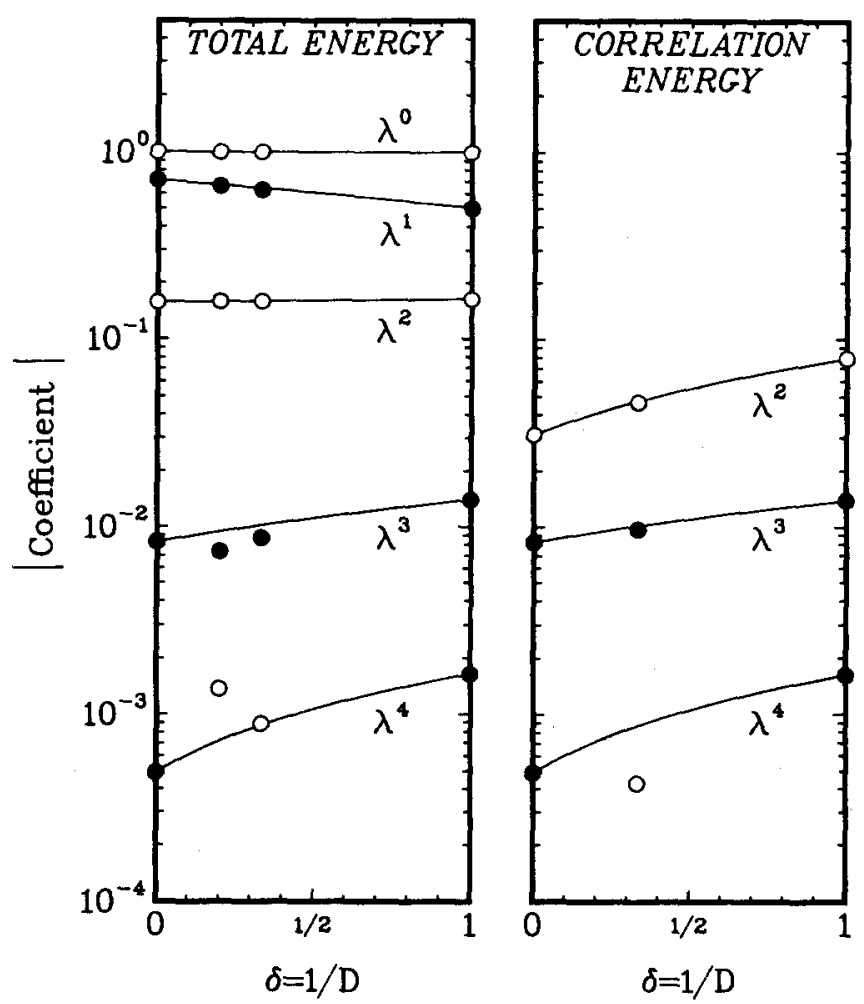

FIG. 6. Perturbation analysis of the dimension-dependence of the total and correlation energies of two-electron atoms. The nonvanishing coefficients through fourth order in $\lambda=1 / Z$ are plotted. Filled and open circles denote positive and negative coefficients, respectively. Solid lines indicate values obtained by linear interpolation between the $D \rightarrow \infty$ and $D \rightarrow 1$ limits (Table II). Absence of the first two terms $\left(\lambda^{0}\right.$ and $\left.\lambda^{1}\right)$ and a high degree of linearity in the next two $\left(\lambda^{2}\right.$ and $\left.\lambda^{3}\right)$ help to account for the observed linearity of the correlation energy.

At order $\lambda^{2}$, which is the lowest order for the CE, the coefficients appear to behave quite linearly. At $D=3$ there is agreement to the nearest 0.001 between exact and interpolated values for all three energies. At order $\lambda^{3}$, this kind of agreement is found for the CE, but not quite for the exact and HF energies. There is apparently again some cancellation taking place. At higher orders in the perturbation expansion there is no sign of interpolability in the coefficients at all; not even the signs are predicted correctly.

Although the linearity of individual coefficients extends at most to third order in $\lambda$, the first few terms of the expan- sion dominate the behavior, and it may be checked that the coefficient behavior suggested by the limited data in Fig. 6 is quite consistent with the more detailed results of the preceding figures. Therefore the observed linearity in $\delta$ of the CE may be attributed to that of its $\lambda^{2}$ and $\lambda^{3}$ terms.

We do not yet have a complete explanation for the regularity of these lowest-order coefficients, but can make a couple of observations which may help in providing one. First, the two terms in question (second and third order in the energy) are those which arise from the first-order correction to the wave function. In particular, for the CE they arise from the first-order error in the HF wave function (which was exact at zeroth order). Second, we note that at the dynamical level these terms constitute the lowest-order effect of the fluctuation potential, which is essentially the difference between the exact (instantaneous) and HF (averaged) forms of the interelectron repulsion. ${ }^{22}$ It may be relevant that the fluctuation potential, unlike the Coulomb potentials from which it is derived, is of relatively short range.

\section{B. Symmetry breaking}

Examination of the perturbation expansions in $\lambda$ can also give some insight into the partial breakdown of linearity in the domain of weakly-bound state (large $D$ and small $Z$ ). It was pointed out that this could be attributed at least in part to the effects of the symmetry breaking at $Z \doteq 1.23$, where the $D \rightarrow \infty$ classical limit switches from a symmetric to an asymmetric structure. A sharp transition occurs only at the point $(\delta, \lambda)=\left(0, \lambda_{0}\right)$, but an examination of the perturbation series reveals how the effects are felt elsewhere.

In Fig. 7 the $\lambda$ perturbation expansions for the $C E$ are plotted through 20th order for several values of $D$. For the dimensional limits the coefficients were obtained from the formulas in Sec. II (explicit values through order $\lambda^{5}$ are given in Table II). For $D=3$ the coefficients were obtained from numerically determined exact ${ }^{23}$ and Hartree-Fock ${ }^{24,25}$ series (Table III); the HF series was extrapolated from fifth to 20 th order. For large $D$ we have simply utilized the expansion $^{15,17}$ for the $1 / D$ term (Table IV); because this converges so poorly compared to the $D \rightarrow \infty$ limit, it will completely dominate the expansion for any large but finite $D$.

From Fig. 7 we see that in general the convergence gets worse as the dimensionality is increased, except that the

TABLE II. Expansions in $\lambda=1 / Z$ for dimensional limit energies. ${ }^{a}$

\begin{tabular}{lrlllll}
\hline \hline & \multicolumn{1}{c}{$\lambda^{0}$} & \multicolumn{1}{c}{$\lambda^{1}$} & \multicolumn{1}{c}{$\lambda^{2}$} & \multicolumn{1}{c}{$\lambda^{3}$} & \multicolumn{1}{c}{$\lambda^{4}$} & \multicolumn{1}{c}{$\lambda^{3}$} \\
\hline$\epsilon_{\infty}$ & -1.0 & 0.707107 & -0.15625 & 0.008286 & 0.000488 & 0.000016 \\
$\epsilon_{\infty}^{\mathrm{HF}}$ & -1.0 & 0.707107 & -0.125 & 0.0 & 0.0 & 0.0 \\
$\Delta \epsilon_{\infty}$ & 0.0 & 0.0 & -0.03125 & 0.008286 & 0.000488 & 0.000016 \\
$\epsilon_{1}$ & -1.0 & 0.5 & -0.162793 & 0.013989 & 0.001643 & 0.000017 \\
$\epsilon_{1}^{\mathrm{HF}}$ & -1.0 & 0.5 & -0.083333 & 0.0 & 0.0 & 0.0 \\
$\Delta \epsilon_{1}$ & 0.0 & 0.0 & -0.079460 & 0.013989 & 0.001643 & 0.000017 \\
\hline \hline
\end{tabular}

Units are $[2 Z /(D-1)]^{2}$ hartree. The series for $\epsilon_{\infty}$ was obtained by expanding Eq. (3). That for $\epsilon_{1}$ was taken from Rosenthal, Ref. 19. The HF coefficients are given directly by Eqs. (4) and (7). 
TABLE III. Expansions in $\lambda=1 / Z$ for exact and interpolated $D=3$ energies. $^{2}$

\begin{tabular}{lrlrrrr}
\hline \hline & $\lambda^{0}$ & \multicolumn{1}{c}{$\lambda^{1}$} & \multicolumn{1}{c}{$\lambda^{2}$} & \multicolumn{1}{c}{$\lambda^{3}$} & \multicolumn{1}{c}{$\lambda^{4}$} & \multicolumn{1}{c}{$\lambda^{5}$} \\
\hline$\epsilon_{3}$ & -1.0 & 0.625 & -0.157666 & 0.008699 & -0.000889 & -0.001036 \\
$\epsilon_{3}^{H F}$ & -1.0 & 0.625 & -0.111003 & -0.001055 & -0.000460 & -0.000208 \\
$\Delta \epsilon_{3}$ & 0.0 & 0.0 & -0.046663 & 0.009754 & -0.000429 & -0.000828 \\
$\epsilon_{\mathrm{LI}}^{\mathrm{HF}}$ & -1.0 & 0.638071 & -0.158431 & 0.010187 & 0.000873 & 0.000016 \\
$\epsilon_{\mathrm{LI}}$ & -1.0 & 0.638071 & -0.111111 & 0.0 & 0.0 & 0.0 \\
$\Delta \epsilon_{\mathrm{LI}}$ & 0.0 & 0.0 & -0.047320 & 0.010187 & 0.000873 & 0.000016 \\
\hline
\end{tabular}

aThe series for $\epsilon_{3}$ was computed by Midtdal (Ref. 23), and that for $\epsilon_{3}^{\mathrm{HF}}$ by Linderberg (Ref. 24) and Coulson and Hibbert (Ref. 25). Coefficients for $\epsilon_{\mathrm{LI}}$ and $\epsilon_{\mathrm{LI}}^{\mathrm{HF}}$ were obtained by interpolating linearly in $1 / D$ between the corresponding quantities in Table II.

$D \rightarrow \infty$ limit itself yields the most well-behaved series of all. The convergence properties of each series are determined by the distance from the origin to the nearest singularity $\lambda *$ in the complex $\lambda$ plane. Using $|\lambda *|=10^{-m}$, where $m$ is the slope of the large-order tail of the series, one finds that this distance is roughly 11.3 for $D \rightarrow \infty, 2.7$ for $D \rightarrow 1,1.1$ for $D=3$, and 0.8 for large but finite $D$. The last of these is actually $\lambda_{0} \doteq 0.81439$, the point of symmetry breaking. ${ }^{15}$ The poor convergence at large $D$ is due to the proximity of this singularity to the origin.

If one considers only the dimensional limits and the real world $(D=1,3, \infty)$, as we did earlier in this section, then the large-order behavior of the series is not very important. This is because the $\mathrm{CE}$ is determined primarily by the lowestorder terms, at least for typical values of the nuclear charge, and these fall outside the asymptotic regime. One can see from Fig. 7, however, that for large $D$ the asymptotic behavior sets in already at low order. In fact, it gives the second nonvanishing term $\left(\lambda^{3}\right)$ the "wrong" sign. It may be checked that the effect of this is to give CEs at high $D$ and low $Z$ which are slightly larger in magnitude than one might expect, based on values elsewhere. This is precisely what was observed in Fig. 3.

\section{APPLICATIONS}

There is important and interesting physics underlying the irregularities we have been discussing, but we repeat that on an absolute scale they remain small. The CE is still a highly linear function of $\delta=1 / D$. In this section we utilize this linearity to motivate two simple procedures for obtaining real-world results from the dimensional limits.

\section{A. Linear interpolation}

The first procedure is linear interpolation (LI) between the $D \rightarrow \infty$ and $D \rightarrow 1$ limiting values. This corresponds precisely to neglecting the nonlinearities plotted in Figs. 3 and 5 , since these were defined as the vertical deviations from straight lines drawn between the two limits. For the CE this procedure gives

$$
\begin{aligned}
\Delta \epsilon_{\mathrm{LI}} & =(1-\delta) \Delta \epsilon_{\infty}+\delta \Delta \epsilon_{1} \\
& =(1-\delta)\left(\epsilon_{\infty}-\epsilon_{\infty}^{\mathrm{HF}}\right)+\delta\left(\epsilon_{1}-\epsilon_{1}^{\mathrm{HF}}\right),
\end{aligned}
$$

where the four limit values are given in Eqs. (3)-(7).

Linear interpolation predicts real two-electron atom CEs to within about $1 \%$. This does not compare with those obtained from the best Hartree-Fock ${ }^{26}$ and Hylleraas-Pe-

TABLE IV. Expansions in $\lambda=1 / Z$ for the first two terms in the $1 / D$ expansion."

\begin{tabular}{lcccccc}
\hline \hline & $\lambda^{0}$ & $\lambda^{1}$ & $\lambda^{2}$ & $\lambda^{3}$ & $\lambda^{4}$ & $\lambda^{5}$ \\
\hline$\epsilon_{\infty}$ & -1 & $\frac{1}{\sqrt{2}}$ & $-\frac{5}{32}$ & $\frac{3}{256 \sqrt{2}}$ & $\frac{1}{2048}$ & $\frac{3}{131072 \sqrt{2}}$ \\
$\epsilon_{\infty}^{\mathrm{HF}}$ & -1 & $\frac{1}{\sqrt{2}}$ & $-\frac{1}{8}$ & 0 & 0 & 0 \\
$\Delta \epsilon_{\infty}$ & 0 & 0 & $-\frac{1}{32}$ & $\frac{3}{256 \sqrt{2}}$ & $\frac{1}{2048}$ & $\frac{3}{131072 \sqrt{2}}$ \\
$\epsilon_{1 / D}$ & 0 & $-\frac{3}{8 \sqrt{2}}$ & $-\frac{31}{1536}$ & $-\frac{1015}{24576 \sqrt{2}}$ & $-\frac{990383}{28311552}$ & $-\frac{21135161}{452984832 \sqrt{2}}$ \\
$\epsilon_{1 / D}^{\mathrm{HF}}$ & 0 & $-\frac{3}{8 \sqrt{2}}$ & $\frac{3}{128}$ & $-\frac{27}{1024 \sqrt{2}}$ & $-\frac{837}{65536}$ & $-\frac{6669}{524288 \sqrt{2}}$ \\
$\Delta \epsilon_{1 / D}$ & 0 & 0 & $-\frac{67}{1536}$ & $-\frac{367}{24576 \sqrt{2}}$ & $-\frac{628799}{28311552}$ & $-\frac{15373145}{452984832 \sqrt{2}}$ \\
\hline \hline
\end{tabular}

-The series for $\epsilon_{\infty}$ and $\epsilon_{\infty}^{\mathrm{HF}}$ were obtained from Eqs. (3) and (4). That for $\epsilon_{1 / D}$ comes from Ref. 15, and for $\epsilon_{1 / D}^{\mathrm{HF}}$ from Ref. 17. 


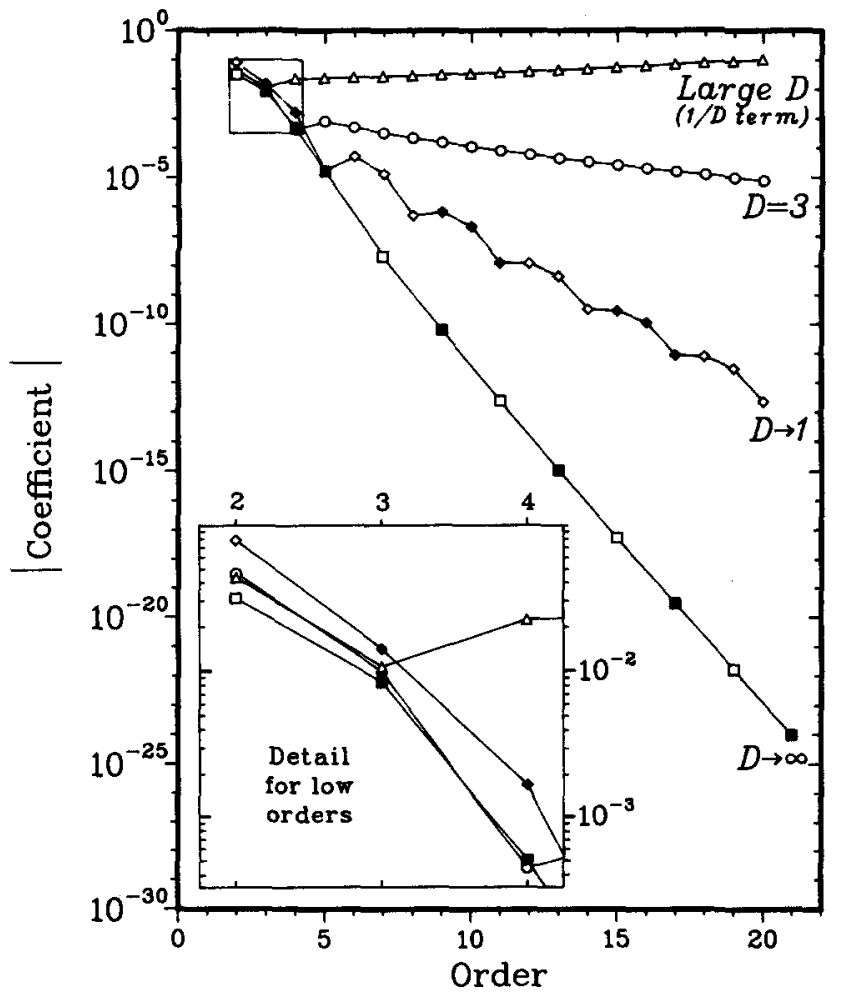

FIG. 7. Perturbation series coefficients through 20th order for several values of $D$. Filled and open symbols again denote positive and negative coefficients, respectively. The convergence properties of each series are determined by the singularity in the complex $\lambda$ plane closest to the origin; the closer the singularity, the worse the convergence. For large but finite $D$ the poor convergence results from the symmetry breaking at $\lambda_{0}=0.81439$.

keris $^{27}$ calculations, which are several orders of magnitude more accurate. These calculations, however, are atypical. Only for a handful of systems (e.g., $\mathrm{Li}, \mathrm{Be}$, and $\mathrm{H}_{2}$ ) are CEs known well enough to talk meaningfully about $1 \%$ accuracy. The very high accuracy achieved for two-electron atoms results from unique features: these systems are the simplest for which a CE can be defined, and benefit from special methods that have been extensively developed. ${ }^{27}$

Because the methods discussed in this paper are not limited by these features, we make comparison to generally applicable methods of calculating $\mathrm{CEs},{ }^{28}$ and in particular to configuration interaction (CI). In Fig. 8 the best available $\mathrm{CI}$ energies for two-electron atoms ${ }^{29-35}$ are compared with those predicted by linear interpolation. In general the two methods give results of comparable accuracy. For positive ions $(\lambda<1 / 2)$ the interpolated energies are more accurate, although unlike those given by CI they are not upper bounds. For negative ions $(\lambda>1 / 2)$ the interpolation method becomes less accurate. For the neutral atom $(\lambda=1 / 2)$ both the interpolated value and the best $\mathrm{CI}$ calculation are very close to the true energy. In the case of $\mathrm{CI}$ this is the result of great computational effort, ${ }^{35}$ while in the case of LI it may be regarded as fortuitous.

\section{B. Planar extrapolation}

For the second method of obtaining real-world CEs we utilize the behavior only at and near $D \rightarrow \infty$. Instead of interpolating linearly between two values (the classical limit

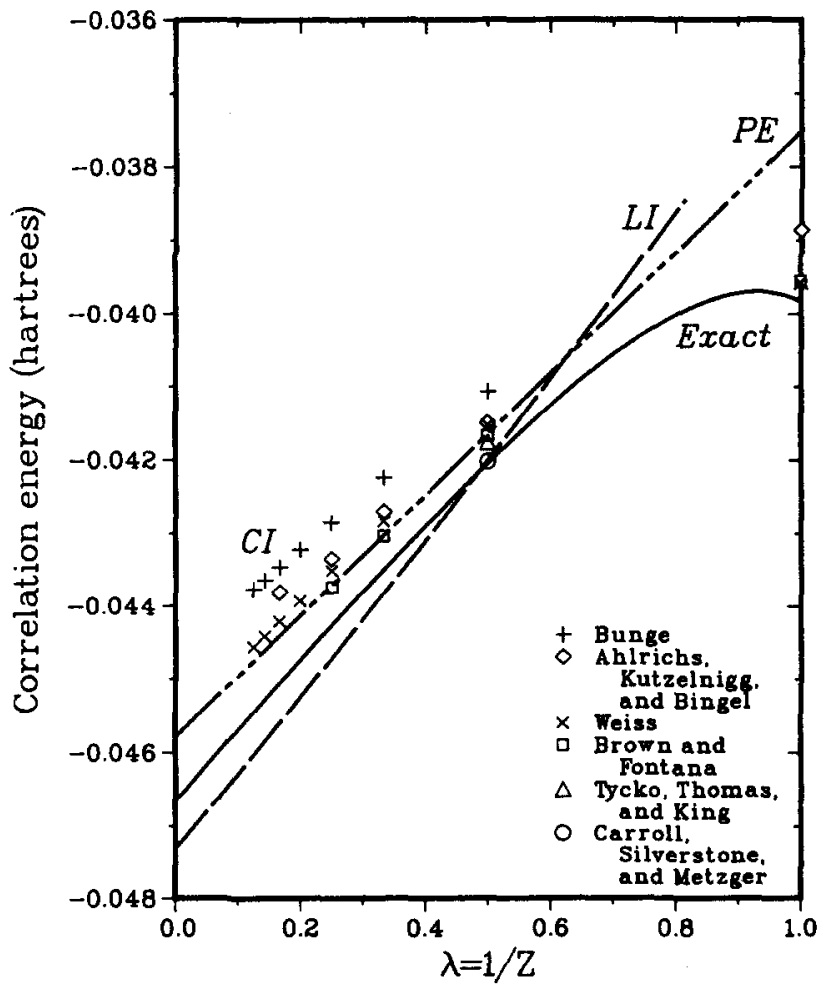

FIG. 8. Correlation energies of real two-electron atoms obtained by configuration interaction (CI), by linear interpolation (LI), by planar extrapolation (PE), and by essentially exact means. All CI calculations on ions and the two best calculations on the neutral atom are plotted. LI values were obtained from Eq. (9), and PE values from Eq. (10). Advantages of PE over LI are its wider applicability, smaller maximum errors, and even greater simplicity.

$D \rightarrow \infty$ and the hyperquantum limit $D \rightarrow 1$ ), we now extrapolate linearly using a value and a slope (the classical limit $D \rightarrow \infty$ and the semiclassical correction term $1 / D$ ). As just described, the method would be simply the $1 / D$ expansion carried to first order. This method, which will be discussed in detail below, gives fairly poor results because the symmetry breaking imposes a small radius of convergence on the $1 /$ $D$ term. We now show how this problem can be avoided.

In general $1 / D$ series are asymptotic. The best approximation to the sum of such a series is obtained by truncating it just before the term of smallest magnitude. If we write the CE formally as a double sum, $\Delta \epsilon=\Sigma c_{m n} \delta^{m} \lambda^{n}$, we see that we can obtain two results, depending on which variable is summed first. Because the series is not convergent in $\delta$, the two results will in general be different.

The usual method of $1 / D$ expansions corresponds to summing on $\lambda$ first. This is because each $\lambda$ expansion is individually convergent, and therefore gets summed to infinte order. Problems arise when this happens, because the full effects of the high-order "tails" of the $\lambda$ expansions, like that of the $1 / D$ term in Fig. 7, are present in the final result. The quantitative effects of this inclusion will be demonstrated below.

By summing on $\delta$ first the tails of the $\lambda$ expansions are cut out. In fact, for all but the lowest two orders in $\lambda$, the $1 / D$ series diverges from the very first term (as is strongly suggested by Fig. 7), and the best approximation to the sum is zero. Furthermore, numerically determined $1 / D$ series $^{36}$ in- 
dicate that at lowest order two terms survive, and at the next order only one. Referring to Table IV, we therefore find that the optimally truncated approximation to the CE is simply

$$
\Delta \epsilon_{\mathrm{PE}}=\left[-\frac{1}{32}+\frac{3}{256 \sqrt{2}} \lambda-\frac{67}{1536} \delta\right] \lambda^{2} \text {. }
$$

The designation "PE" stands for planar extrapolation, because this truncation corresponds to assuming that the surface plotted in Fig. 1 may be approximated by a plane determined by the behavior at the origin. In fact, Eq. (10) describes the plane tangent to the true energy surface at the origin. Real-world CEs predicted by such planar extrapolation are compared to $\mathrm{CI}$ and $\mathrm{LI}$ values in Fig. 8. Once again the accuracy obtained is comparable to that of $\mathrm{CI}$.

Comparing now the two dimensional continuation methods discussed so far, we see in Fig. 8 that overall they achieve similar accuracy. However, PE has several advantages over LI. First, PE is immediately applicable to the full range of two-electron atoms, whereas $L I$ is presently limited by Eq. (3) to atoms which have symmetric classical limits. Second, the maximum errors are less for PE. And third, PE is simpler; among other things, this means that it may generalize more easily. (The PE values also seem to give an upper bound, but there is no apparent reason for this.)

We caution that not enough terms of the perturbation expansions are known to say for sure what the optimal asymptotic truncation is. We can only say that on the basis of limited data Eq. (10) appears to be it, at least for $D=3$. Actually, adding a term or two from Table IV does not change the qualitative agreement with experiment-in fact some terms even help. The important point is that the adverse effects of the high-order tails of the expansions are excluded by this method. We now consider what happens when they are not.

\section{1/D expansions}

The straightforward calculation of the classical limit $D \rightarrow \infty$ and semiclassical correction terms in powers of $1 / D$, without regard to convergence until the final summation, gives the usual $1 / D$ expansion. ${ }^{9-12}$ Formally, this approach corresponds to reversing the order of summation in the double sum over $\delta$ and $\lambda$.

The first three partial sums of the $1 / D$ expansion are plotted in Fig. 9, along with several reference curves. The one-term sum is just the $D \rightarrow \infty$ limit itself, while the next two partial sums were obtained by adding in turn the firstand second-order semiclassical corrections to that limit. In the figure the optimal asymptotic truncation, which is the partial sum obtained by truncating the series before the term smallest in magnitude, is indicated by a heavier line.

A couple of features of the $1 / D$ expansion mentioned above are apparent in Fig. 9. First is its asymptotic character. Depending on the nuclear charge either the first or second partial sum gives the best energy; the third- and higherorder partial sums are uniformly worse. Second is the profound effect of the symmetry breaking on the $1 / D$ term. Although the square root branch point lies far off-scale, its effects are apparent. Except at high $Z$, it renders the 1/D expansion useless for predictive purposes. ${ }^{13}$

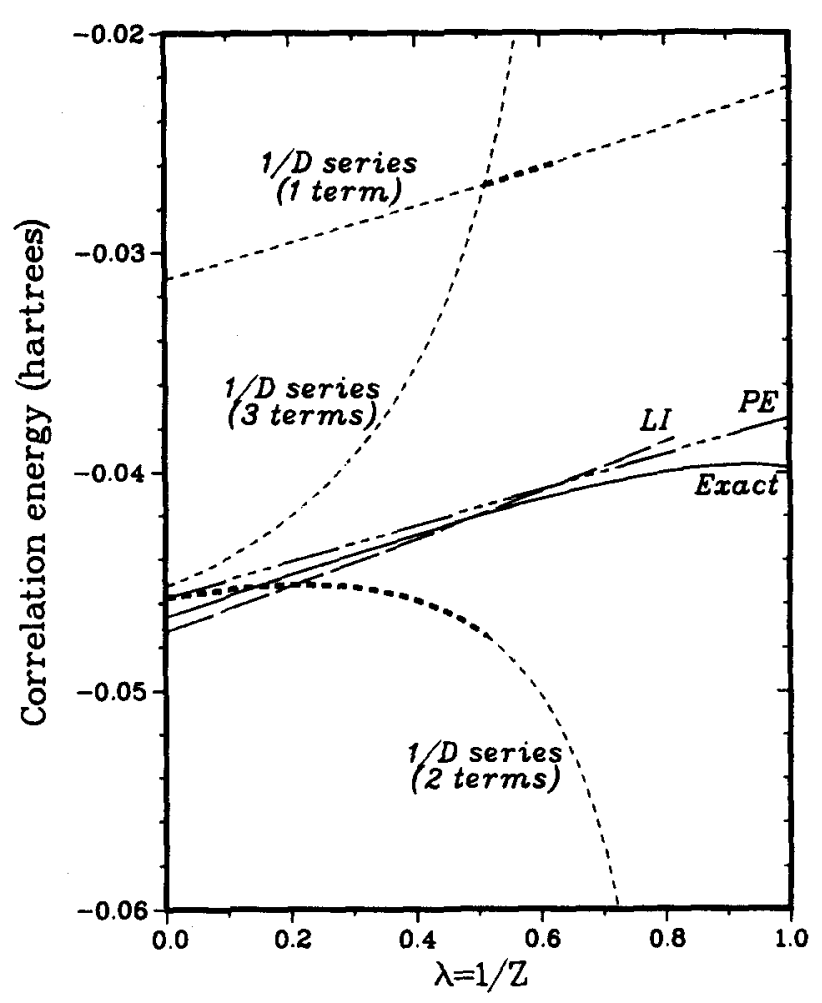

FIG. 9. Correlation energies obtained from the $1 / D$ expansion at several orders, along with more accurate results for comparison. The optimal asymptotic approximation at each value of $Z$ is indicated by the heaviest line. The $1 / D$ expansion performs poorly because the transition from a symmetric to an asymmetric classical limit structure at $\lambda_{0} \doteq 0.81439$ renders the semiclassical expansion nonanalytic there. $L I$ and $P E$ are similar in spirit to the $1 / D$ expansion, but work much better because each in its own way avoids the disruptive effects of the symmetry breaking.

Roughly speaking, the semiclassical $1 / D$ expansion fails because of refractory behavior associated with the region of small $Z$ and large but finite $D$. The success of the two alternative approaches discussed earlier in this section may be attributed to the fact that each avoids this region in its own way. Thus, the linear interpolation method avoids the effects of large but finite $D$ by utilizing only the two dimensional limits $D \rightarrow \infty$ and $D \rightarrow 1$. The planar extrapolation method, on the other hand, avoids the effects of small $Z$ by truncating the $1 /$ $Z$ expansion at low order.

\section{DISCUSSION}

The stimulus for this work was a review of $1 / D$ expansions which utilized the two-electron atom as an example. ${ }^{9}$ In the usual form, the expansion gave poor results for the total energy, but it was found ${ }^{15}$ that rescaling and interpolation using the known $D \rightarrow 1$ limit greatly improved the accuracy. As we have just seen, the correlation energy serves as a much more stringent test, and even the rescaled $1 / D$ expansion works poorly here. The difficulty lies in the semiclassical portion of the expansion, which becomes singular at $\lambda_{0} \doteq 0.81439$ because of the transition to an asymmetric classical limit structure.

Nevertheless, the CE is a remarkably well-behaved function. Numerical calculations reveal that it varies almost linearly with $\delta=1 / D$ over the range $1<D<\infty$. Some char- 
acteristic irregularities arising from the symmetry breaking can be seen on close examination, especially in the vicinity of the transition (large $D$ and small $Z$ ), but these effects are small on an absolute scale. The overall regularity of the behavior suggests that $1 / D$ expansions may not be the most effective way to utilize dimensional continuation. In this paper we have introduced two alternative methods which, like the $1 / D$ expansion, utilize only easily calculated dimensional limit quantities, but yield far more accurate real-world predictions. The first method (linear interpolation) utilizes only the classical limit $D \rightarrow \infty$ and the hyperquantum limit $D \rightarrow 1$, thereby avoiding entirely the troublesome semiclassical expansion in powers of $1 / D$. The even simpler second method (planar extrapolation) utilizes the $1 / D$ expansion as a starting point, but by asymptotically truncating at each order of $1 / Z$ it excises the undesirable effects of the symmetry-breaking transition. Both methods give CEs accurate to about $1 \%$.

For any system other than a two-electron atom this kind of accuracy would almost certainly exceed that currently available, because methods which are generally applicable to the calculation of CEs converge extremely slowly. Some partial results for larger atoms indicate that the generalization of the methods described in $\mathrm{Sec}$. $\mathrm{V}$ will be both accessible and accurate. ${ }^{37}$ So far only the $D \rightarrow \infty$ limit (the term which the two methods have in common) has been calculated fully, but it is clear that the $1 / D$ term can be calculated explicitly as well. The $D \rightarrow \infty$ limit by itself can be used to obtain predictions for $D=3$ exact and Hartree-Fock energies which are accurate to a few percent for all atoms. As in the two-electron case, however, there is a high degree of cancellation between the exact and HF errors, so that CEs are predicted much more accurately. Typically they are underestimated by $0-2 \mathrm{eV}$. For second-row atoms, which are the largest ones for which reasonably accurate reference values are available, one obtains between $90 \%$ and $100 \%$ of the true CEs.

In extending the methods of this paper to larger atoms, the most formidable problem may eventually be not their derivation, but rather their assessment. This will be more difficult for larger atoms for several reasons. First is the unavailability of calculations except for $D=1,3, \infty$. This will make it hard to quantify linearity. Second is the fact that for large atoms not even $D=3$ correlation energies are available. Reasonably accurate $( \pm 10 \%)$ experimental values are available only through argon, and theoretical values only through neon. Finally, there is the fact that $1 / Z$ expansions like those used in Sec. IV have been calculated only for twoelectron atoms.

The two-electron atom is the simplest many-body system, and as such has always served as a test case for electronic structure methods. It is the natural first step in the application of dimensional continuation methods. The second step, already underway, is the application of these methods to a range of more complex systems which have already been studied by conventional means. This will give some idea of what can be expected in general from dimensional continuation methods. If they prove successful here, then the third step will be their application to systems for which other methods have proven too difficult to apply, or have failed. The simplicity of the new approach makes this a realistic prospect.

'R. McWeeny, in The New World of Quantum Chemistry, edited by B. Pullman and R. Parr (Reidel, Dordrecht, 1976), p. 3.

${ }^{2} \mathrm{O}$. Sinanoğlu and K. A. Brueckner, Three Approaches to Electron Correlation in Atoms (Yale, New Haven, 1970), p. 79.

${ }^{3}$ E. Clementi and C. Roetti, At. Data Nucl. Data Tables 14, 177 (1974); A D. McLean and R. S. McLean, ibid. 26, 197 (1981); J. G. Snijders, P. Vernooijs, and E. J. Baerends, ibid. 26, 483 (1981).

${ }^{4}$ F. Sasaki and M. Yoshimine, Phys. Rev. A 9, 17 (1974).

${ }^{5}$ E. Clementi, J. Chem. Phys. 39, 175 (1963); IBM J. Res. Develop. 9, 2 (1965); A. Veillard and E. Clementi, J. Chem. Phys. 49, 2415 (1968).

${ }^{6} \mathrm{R}$. D. Cowan, The Theory of Atomic Structure and Spectra (University of California, Berkeley, 1981), p. 204.

${ }^{7}$ M. Gell-Mann and K. A. Brueckner, Phys. Rev. 106, 364 (1957).

${ }^{8}$ See Ref. 2, p. 180.

${ }^{9}$ E. Witten, Physics Today 33, (No. 7) 38 (1980).

${ }^{10}$ L. D. Mlodinow and N. Papanicolaou, Ann. Phys. (NY) 131, 1 (1981).

"P. du T. van der Merwe, J. Chem. Phys. 81, 5976 (1984).

${ }^{12} \mathrm{O}$. Goscinski and V. Mujica, Int. J. Quantum Chem. 29, 897 (1986).

${ }^{13}$ J. G. Loeser and D. R. Herschbach, J. Phys. Chem. 89, 3444 (1985).

${ }^{14}$ D. J. Doren and D. R. Herschbach, Chem. Phys. Lett. 118, 115 (1985).

${ }^{15}$ D. R. Herschbach, J. Chem. Phys. 84, 838 (1986).

${ }^{16}$ J. G. Loeser and D. R. Herschbach, J. Chem. Phys. 84, 3882, 3893 (1986).

${ }^{17}$ D. Z. Goodson and D. R. Herschbach, J. Chem. Phys. (to be published).

${ }^{18}$ D. R. Herrick and F. H. Stillinger, Phys. Rev. A 11, 42 (1975).

${ }^{19} \mathrm{C}$. M. Rosenthal, J. Chem. Phys. 55, 2474 (1971).

${ }^{20}$ Y. Nogami, M. Vallières, and W. van Dijk, Am. J. Phys. 44, 886 (1976); L. L. Foldy, ibid. 44, 1192 (1976).

${ }^{21}$ E. Clementi, J. Chem. Phys. 38, 2248 (1963).

${ }^{22}$ O. Sinanoğlu, Proc. Natl. Acad. Sci. U.S.A. 47, 1217 (1961); R. S. Berry, J. Chem. Ed. 43, 283 (1966).

${ }^{23}$ J. Midtdal, Phys. Rev. 138, A1010 (1965).

${ }^{24}$ J. Linderberg, Phys. Rev, 121, 816 (1961).

${ }^{25}$ C. A. Coulson and A. Hibbert, Proc. Phys. Soc. 91,33 (1967); A. Hibbert, ibid. 91,819 (1967).

${ }^{26}$ J. L. Gázquez and H. J. Silverstone, J. Chem. Phys. 67, 1887 (1977).

${ }^{27}$ D. E. Freund, B. D. Huxtable, and J. D. Morgan III, Phys. Rev. A 29, 980 (1984).

${ }^{28}$ A. Hibbert, in Progress in Atomic Spectroscopy, edited by W. Hanle and H. Kleinpoppen (Plenum, New York, 1978), p. 1.

${ }^{29}$ C. F. Bunge, Theor. Chim. Acta 16, 126 (1970)

${ }^{30}$ R. Ahlrichs, W. Kutzelnigg, and W. A. Bingel, Theor. Chim. Acta 5, 289 (1966).

${ }^{31}$ A. W. Weiss, Phys. Rev. 122, 1826 (1961).

${ }^{32}$ R. T. Brown and P. R. Fontana, J. Chem. Phys. 45, 4248 (1966).

${ }^{33}$ D. H. Tycko, L. H. Thomas, and K. M. King, Phys. Rev. 109, 369 (1958).

${ }^{34}$ D. P. Carroll, H. J. Silverstone, and R. M. Metzger, J. Chem. Phys. 71, 4142 (1979).

${ }^{35}$ D. W. Zaharevitz, H. J. Silverstone, and D. M. Silver, in Supercomputer Applications, edited by R. W. Numrich (Plenum, New York, 1985), p. 263.

${ }^{36}$ J. G. Loeser and D. R. Herschbach, J. Chem. Phys. 86, 2114 (1987).

${ }^{37}$ J. G. Loeser, J. Chem. Phys. (to be published). 